\title{
Canales de información y entretenimiento en el metro y en los autobuses de España
}

\author{
Sergio MENA MuÑOZ \\ Universidad Nebrija \\ menasergio@menasergio.es \\ Luis Miguel MARTínEZ FERNÁNDEZ \\ Universidad Complutense de Madrid \\ lesmes@ccinf.ucm.es
}

Recibido: $15 / 03 / 2011$

Aceptado: 26/07/2011

\begin{abstract}
Resumen Madrid y Barcelona y en los autobuses urbanos de algunas ciudades españolas. televisión.

\section{Information and Entertainment Channels at Underground and Buses Systems in Spain}

Las posibilidades del desarrollo de las tecnologías de la información han hecho posible la unión de dos ámbitos tan dispares como los medios de transporte y los medios de comunicación. Ya es posible disfrutar de un canal de televisión sentados en el asiento de los transportes públicos, algo impensable hace veinte años. Se abre así un campo lleno de posibilidades para los gestores, los responsables de contenidos y los publicistas, que tienen a su disposición una audiencia cautiva nunca antes considerada que puede rebelarse contra la injerecia de su espacio público. En este trabajo se investigan las características, las posibilidades y el impacto producido por la puesta en marcha de dos canales multimedia en el metro de

Palabras clave: Canales Multimedia, medios de transporte, audiencia cautiva, espacios públicos,

\begin{abstract}
The possibilities of development of information technologies have made possible the union of two such disparate areas as transportation and media. Now it is possible to enjoy a television channel sitting on the seat of public transport, something unthinkable twenty years ago. This opens up a field full of possibilities for managers, responsibles for the content and for advertisers, who have at their disposal a captive audience never before considered who may rebel against the interference of their public space. This article researches the characteristics, possibilities and the impact produced by the launch of two media channels in Madrid and Barcelona Underground and city buses in some Spanish cities.

Keywords: Multimedia Channels, Transportation, captive audience, public spaces, television

\section{Referencia normalizada}

MENA MUÑOZ, Sergio y MARTÍNEZ FERNÁNDEZ, Luis Miguel (2011): “Canales de información y entretenimiento en el metro y en los autobuses de España". Estudios sobre el mensaje periodístico, vol. 17, núm. 2, págs.: 531-548. Madrid, Servicio de Publicaciones de la Universidad Complutense.

Sumario: 1. Introducción. 1.1. Hipótesis de partida. 2. Estado de la cuestión. 3. Investigación sobre programación. 3.1. Metodología. 3.2. Análisis de los resultados. 4. Investigación sobre el Efecto de conformidad. 4.1. Metodología. 4.2. Análisis de los resultados. 5. Investigación sobre niveles sonoros en los ferrocarriles metropolitanos. 5.1. Metodología. 5.2. Análisis de los datos. 5.2.1. La interferencia del sonido. 6. Investigación sobre la respuesta de la audiencia. 6.1. Metodología. 6.2. Análisis de los resultados del canal Metro. 6.2.1. Preferencias de los usuarios. 6.3. Análisis de los resultados del canal Bussi. 7. Propuesta de modelo comunicacional para un canal en los transportes públicos. 8. Conclusión. 9. Referencias bibliográficas.
\end{abstract}




\section{Introducción}

Entre los años 2000 y 2007 se pusieron en marcha y se desarrollaron en una primera etapa en España dos iniciativas pioneras en Europa que han aunado los medios de comunicación con los transportes públicos. La empresa Bussitel, de Valencia, comenzó a emitir una serie de contenidos audiovisuales en las flotas de los autobuses urbanos de la propia Valencia, además de Sevilla y San Sebastián.

Por otro lado, la compañía TvTrans hizo lo mismo dentro de los andenes del Metro de Madrid, de los Ferrocarrils de la Generalitat de Cataluña y del Metro de Barcelona.

Si bien hasta entonces la relación transportes-medios de comunicación se había limitado a mostrar contenidos reproducibles (sobre todo en autocares, trenes y aviones), a partir de la irrupción de estos nuevos canales, ayudados por la evolución de la técnica, se ha conseguido emitir en directo una programación estable y dinámica en los medios de transporte urbanos citados. En el desarrollo de estas dos experiencias innovadoras se centra nuestra investigación.

En un primer momento, el objetivo de los sistemas de información al viajero era proporcionar información estática o dinámica; información que le ayudara a llegar a destino de la forma más eficiente, rápida y sobre todo cómoda (VV.AA., 2009: web), pero poco a poco, con el paso del tiempo, el proceso se ha ido haciendo más complejo.

Los efectos que pueda producir la comunicación de masas entre los usuarios de los medios de transporte es aún un campo poco estudiado. El hecho de que los tiempos muertos de espera y de viaje se hayan convertido en una búsqueda por parte del usuario de un medio de distracción, no es normalmente una condición necesaria y suficiente de efecto sobre la audiencia (BURGUELIN, 1974: 151). Independientemente de ese interés por rellenar espacios vacíos, hay que considerar que el ojo humano se dirige por instinto hacia aquello que se mueve (BASSAT, 2001: 228) y, por tanto, la audiencia cautiva de dentro de un autobús o de un vagón de metro, es más proclive a la recepción de mensajes que la habitual de otros ámbitos.

Los gestores de los transportes públicos que se han estudiado en este trabajo han ido dando mayor importancia a estos medios conforme ha ido pasando el tiempo. Conscientes, sin duda, de que la nueva fórmula supone un aliciente para hacer más atractivos sus servicios, en detrimento de otros transportes, que no cuentan con este tipo de servicio añadido.

Al mismo tiempo, esta experiencia también ayuda en la comunicación corporativa de la empresa y, además, le supone una financiación extra como consecuencia de las inserciones publicitarias. No debemos olvidar que la emisión de estos nuevos canales se dirige a una audiencia amplia y que además permanece en un espacio cerrado donde están "cautivos", lo que provoca una mayor atención a la programación que se les ofrece.

El tiempo del estudio se ha parcelado entre los años 2000 y 2007, espacio que abarca desde los primeros pasos de su implantación en los medios de transporte, hasta el punto de inflexión que supuso el inicio de la crisis (año 2008). En este tiempo, con una inversión tanto en infraestructuras como en publicidad en alza, se ha desarrollado el primer capítulo de una andadura que aún continúa, pero con otras características distintas y que esperamos abordar en otra investigación en el futuro. 


\subsection{Hipótesis de partida}

En la investigación nos planteamos distintas cuestiones en forma de hipótesis de trabajo con respecto a estos canales ubicados en los transportes públicos. Hipótesis referidas a su entorno de emisión, a su naturaleza, a su impacto y a sus contenidos. A saber:

1. Los canales multimedia de los transportes públicos basan su estructura y su programación en los medios de comunicación tradicionales.

2. Los usuarios de los medios de transporte son favorables a la existencia y desarrollo de estos canales.

3. Los usuarios de transportes prefieren que no emitan sonido y que no sean intrusivos.

4. La accesibilidad y usabilidad de estos medios no es completa al no amparar en su naturaleza el acceso a los contenidos de los minusválidos visuales.

En este marco, nuestro objetivo pretende identificar cuál es el papel de estos medios de comunicación dentro del panorama audiovisual español, analizar sus características como medio de comunicación y como canales exclusivos dentro de un ámbito novedoso, conocer el impacto que han producido en el público y proponer, en última instancia, un modelo comunicacional que sirva también para los canales futuros que puedan surgir y que tengan la misma naturaleza.

\section{Estado de la cuestión}

Como ya se ha indicado, dos son las empresas que operan en España estos medios. TvTrans, empresa del grupo Dragados durante el tiempo que ha abarcado nuestras investigaciones, proporciona una programación y una red de pantallas a todos los usuarios del metropolitano madrileño a través de Canal Metro Madrid, en Barcelona a través de Canal Metro Barcelona y en los ferrocarriles catalanes a través de EntreTrenTV. La empresa TvTrans se ha conectado durante el tiempo del estudio a su proveedor de red a través de varias líneas dedicadas con una velocidad de 1 Mbps en sus programaciones de Madrid y Barcelona. En la ciudad condal, en 2005 existían 68 estaciones con el sistema instalado, con una cobertura aproximada del $81 \%$ de la red (VV.AA., 2006: web).

La empresa Bussitel S.A., radicada en Valencia, explota su canal de información urbana con un "revolucionario sistema de comunicación multimedia a bordo del transporte público colectivo" (CORTINA, 2004: E5). Este canal permite a la empresa de transporte comunicarse, además, en tiempo real con los pasajeros para ofrecerles información del servicio y una programación diaria. Este sistema ofrece, además, información sobre la siguiente parada y el estado del tráfico, así como de los lugares turísticos que quedan cerca del recorrido de la línea. Su explotación se ha concentrado durante el tiempo de las investigaciones en tres ciudades: Valencia, Sevilla y San Sebastián.

\section{Investigación sobre programación}

Como ya se ha indicado, uno de los objetivos de esta investigación es analizar las características como medio de comunicación de estos canales instalados en los trans- 
portes públicos y demostrar que basan su estructura y su programación en los medios de comunicación tradicionales. En este sentido, los objetivos concretos que nos hemos planteado han sido los siguientes:

- Averiguar cuál es el orden de emisión de los contenidos de la programación.

- Calcular cuál es el porcentaje de publicidad que se emite en la programación.

- Medir el porcentaje de contenidos que tratan de contenidos informativos y del resto de contenidos.

- Evaluar los cambios sufridos por la programación entre 2004 y 2007.

\subsection{Metodología}

Partimos de una recogida directa de datos en el lugar de emisión de la programación en los dos tipos de canales analizados. En concreto, comparamos los datos recogidos dentro de tres autobuses de la línea 89 (circular) de la EMT de Valencia entre las paradas de Cardenal Benlloch-Dr. Vicente Pallarés y los de la parada de la calle Dr. Peset Aleixandre-Avenida Burjassot. Otro tanto hicimos con los datos recogidos en distintos lugares de emisión de la programación de Canal Metro Madrid, Canal Metro Barcelona y EntreTrenTV.

Cuadro 1: Ámbitos y oleadas de las investigaciones sobre programación

\begin{tabular}{|c|c|c|c|c|}
\hline EntreTrenTV & $\begin{array}{c}\text { Canal Metro } \\
\text { BCN }\end{array}$ & $\begin{array}{c}\text { Canal Metro } \\
\text { Madrid }\end{array}$ & Canal Bussi \\
\hline Lugar & $\begin{array}{c}\text { Estación de Plaça } \\
\text { Catalunya (FGC) }\end{array}$ & $\begin{array}{c}\text { Plaça Catalunya } \\
\text { Estación de } \\
\text { (Metro) }\end{array}$ & $\begin{array}{c}\text { Estación de Ave- } \\
\text { nida de América } \\
\text { Línea 6 (Metro) }\end{array}$ & $\begin{array}{c}\text { Interior bus línea } \\
89 \text { EMT Valencia }\end{array}$ \\
\hline $\begin{array}{c}\text { Duración } \\
\text { muestra }\end{array}$ & $20^{\prime}$ (c) & $10^{\prime}(\mathrm{c})$ & $\begin{array}{c}40^{\prime}(\mathrm{c}) \\
60^{\prime}(\mathrm{d})\end{array}$ & $\begin{array}{c}15^{\prime} \text { (a) } \\
22^{\prime}(\mathrm{b}) \\
15^{\prime}(\mathrm{d})\end{array}$ \\
\hline
\end{tabular}

En el primer caso, el relativo a las pantallas en autobuses urbanos, se realizó la recogida de datos en tres años diferentes: el jueves 16 de marzo de 2004 entre las 12:00 y las 12:15 horas, en un bucle de contenidos de quince minutos de duración (a); el viernes 16 de marzo de 2005 entre las 17:10 y las 17:32 horas (b) y el 16 de marzo de 2007 entre las 17:15 y las 17:30 horas (d). Hay que subrayar que la programación de Canal Bussi es la misma en los tres puntos de emisión de España.

Por la parte del metro, se recogieron los datos en la estación de Avenida de América de Madrid, en el andén de la Línea 6, el jueves 16 de marzo de 2004 entre las 13:00 y las 13:40 horas en un bucle de contenidos de veinticinco minutos de duración (c) y el viernes 16 de marzo de 2007, entre las 13:00 y las 14:00 horas (d). En Barcelona se recogieron datos en la Estación de Plaça de Catalunya, el jueves 16 de marzo de 2004, entre las 13:00 y las 13:20 horas en el andén de FCC y de 13:00 a 13:10 en el andén del metro (c). En la recogida, se anotaron los distintos contenidos de cada programación, así como las pausas comerciales. 


\subsection{Análisis de los resultados}

En el caso de Canal Metro, la publicidad evolucionó de manera positiva. Así, mientras que en 2004 los contenidos publicitarios ocupaban un 17,37\%, al año siguiente doblaba las magnitudes, por encima del $43 \%$. Tal ampliación de publicidad supuso todo un espaldarazo por parte de los inversores al canal, ya que significaba que creían en el proyecto y que había resultados económicos positivos. Es determinante que en sólo un año aumentara tanto el porcentaje $(+25,93 \%)$. Aparentemente, este aumento va en contra de la opinión de los espectadores, que, no olvidemos, constituyen una audiencia cautiva y acepta los anuncios a regañadientes.

Dado el interés comercial de la empresa, hay que subrayar que el diseño de programación está muy ligado a ese aumento de publicidad. Si aumentan los anuncios, se reducen los contenidos de noticias. En este caso, en tan solo doce meses, las noticias perdieron un $9,46 \%$ de cuota. (Ver gráficos 1 y 2 )

Gráficos 1 y 2: Porcentaje de publicidad, noticias y promocionales en la programación de Canal Metro

oleada de 2004

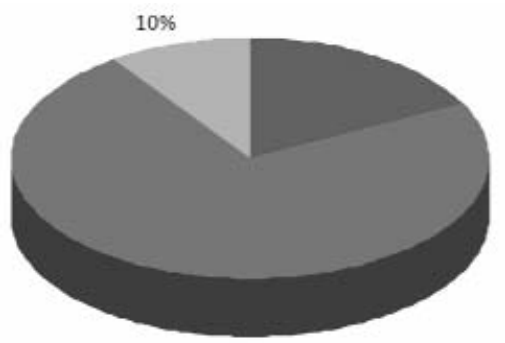

oleada de 2005

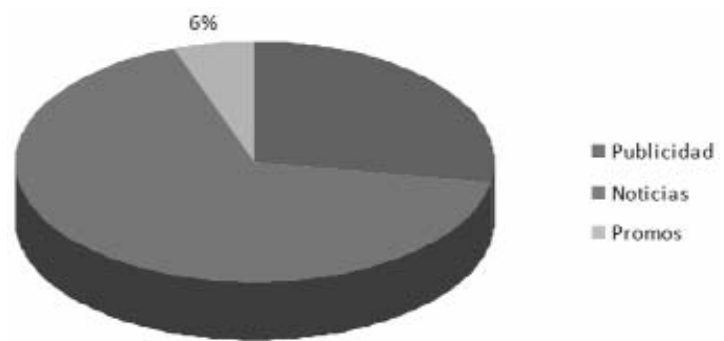

En el caso de Canal Bussi, después de tres oleadas, la tendencia es absolutamente la contraria. En tres años, la inversión publicitaria cayó del 33,3\% en 2004 al 16\% en 2007 (-17,3\%). En cuanto a los contenidos de noticias y entretenimiento, en el mismo periodo de tiempo experimentó un alza del 15\%. En 2004 los contenidos ocupaban un $60 \%$, y en 2007 un $75 \%$.

El diseño de la programación no ha variado nada en tres oleadas realizadas. Las pautas dedicadas a las noticias fueron 44 en 2004 y 50 en 2007, un nivel muy parecido en el que se siguió utilizando el pase con cabeceras entre bloque y bloque.

Por tanto, y como conclusión, podemos decir, que la evolución de la publicidad es positiva en el caso del metro y negativa en el de los autobuses, con más de un 43,3\% de diferencia entre lo que ha aumentado por un lado y lo que ha bajado por otro.

En el caso del resto de contenidos, los correspondientes al metro aumentaron 9 minutos y 42 segundos entre 2004 y 2005 y en los autobuses subieron un $10 \%$ en el mismo periodo. Las estructuras entre los dos medios de comunicación son claramente diferentes. Mientras que en los metros no se han percibido cambios significativos, y se sigue utilizando la flexibilidad como elemento diferenciador, los autobuses también han sido fieles a sus principios y tampoco han experimentado cambios en su estrategia (Ver gráficos 3, 4 y 5) 
Gráficos 3, 4 y 5: Porcentaje de publicidad, noticias y promocionales en la programación de Canal Bussi

oleada de 2004

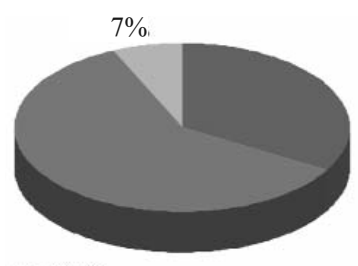

oleada de 2005

$8 \%$

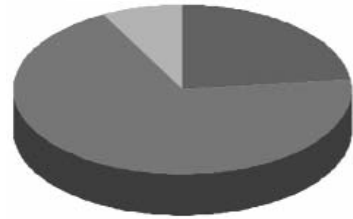

oleada de 2007

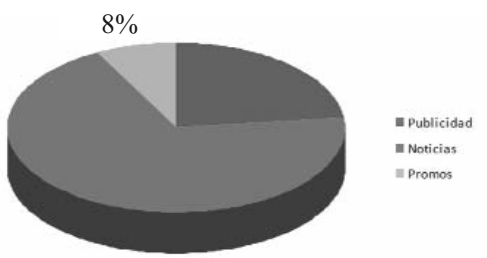

\section{Investigación sobre el Efecto de conformidad}

Tal y como indican SMITH y MACKIE (1997: 357), el efecto de conformidad mide la convergencia de las respuestas individuales hacia las normas del grupo. El hecho de saber por qué el ser humano adopta las posiciones o se comporta de la manera en que lo hace socialmente, ha sido objeto de interés por parte de los investigadores. Existen unos cánones preestablecidos que se nos han inculcado por medio de la cultura del grupo, de la "manada", que es tenida muy en cuenta por los programadores y los publicistas para dar forma a su producto. Uno de los efectos usados en esos cánones es el de conformidad, una manera, por otra parte, de sentirse parte del clan. AscH (1995) realizó varios trabajos de campo en los que demostró que parte de nuestro comportamiento social está propiciado por lo que vemos que hace el resto de personas que nos rodean. Este movimiento psicológico de tendencia social, fue denominado por el mismo autor como "Efecto de conformidad". De esta manera hemos querido adaptar este efecto a uno de los ámbitos objeto de nuestro estudio.

En el andén de una estación de metro, dentro de en un túnel, pocas son las opciones de concentrar nuestra mirada que no sean las pantallas de emisión del canal investigado. Partiendo de esta premisa, hemos querido averiguar cuáles son las partes de la estación donde más se concentra el público; con un triple objetivo:

- Conocer cuáles son las pantallas que más usuarios concentran

- Calcular si las partes de la estación más concurridas coinciden con la colocación de alguna pantalla de emisión

- Evaluar si la colocación de las pantallas obedece a una estrategia premeditada

\subsection{Metodología}

Partimos de una recogida directa de datos de la emisión en la Estación de metro de Moncloa, en el andén de la Línea 6, el 14 de marzo de 2005. La recogida se realizó en un horario comprendido entre las 18:00 y las 18:30 horas, en el que los convoyes pasaron por la estación en intervalos medios de entre 3:30 y 5 minutos. Durante este tiempo, pararon 7 trenes en el Andén 1 y 8 trenes en el Andén 2. Se constituyeron dos equipos de recolección de datos, cada uno responsable de los datos emanados de cada uno de los andenes que componen la estación.

Para ser más precisos en nuestros cálculos, realizamos un croquis a escala gracias a la información gráfica obtenida del Catastro de viviendas. A continuación, identificamos las distintas pantallas que existen en las vías y en los andenes y dibujamos un espacio de influencia de cada una de ellas de 20 metros de diámetro en escala (Ver gráfico 6) 
Gráfico 6: Planta de estudio en cuadrículas y puntos de colocación de pantallas y zonas de influencia en la estación de metro de Moncloa (Línea 6).

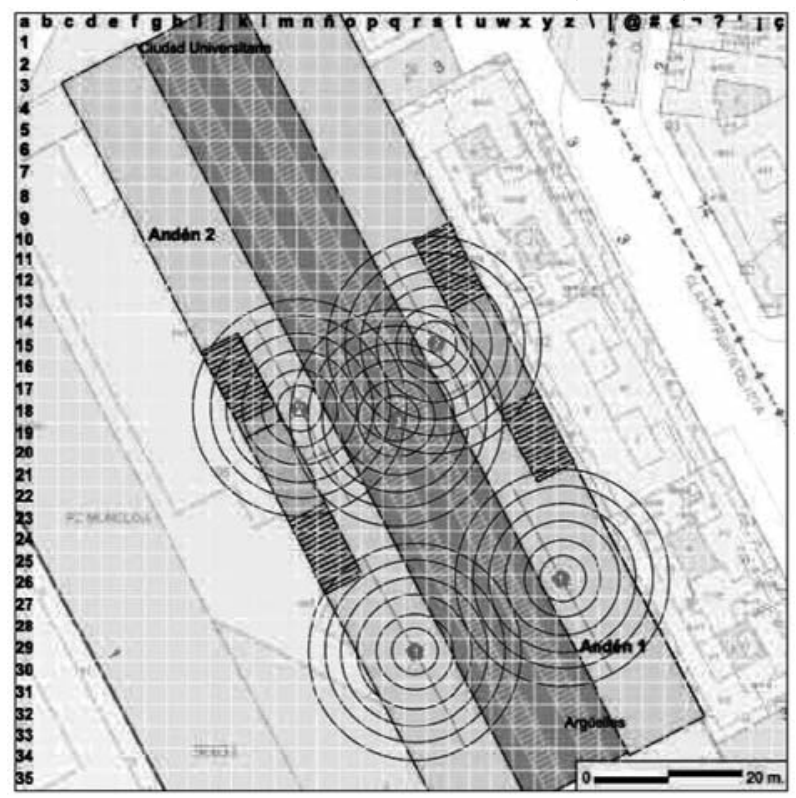

Después se hizo una cuadrícula de la estación con una fila horizontal que recoge todas las letras del abecedario más varios signos gráficos del teclado. Por otro lado, se numeran las filas verticales desde el 1 hasta el 35. Una vez colocadas las cuadrículas sobre los puntos de las pantallas y su área de influencia, se observó que ese área se limita a las cuadrículas (en vertical) de la letra i al signo \# y (en horizontal) del 9 al 34, concentrándose en estas celdas el contenido de nuestros datos (Ver gráfico 7)

Cada cinco minutos se apuntaron en la casilla correspondiente el número de personas que ocuparon su espacio en ese intervalo. La recolección se hizo cada cinco minutos en un período de treinta, con lo que se realizaron seis tablas de recopilación de datos. Las celdas en las que no se han recogido datos porque no se ha parado nadie o porque coincide con un elemento arquitectónico, se han dejado en blanco, pero coinciden con el valor cero.

Gráfico 7: Cuadrícula de recogida de datos

\begin{tabular}{|c|c|c|c|c|c|c|c|c|c|c|c|c|c|c|c|c|c|}
\hline Ti & & & m & & & 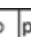 & dq & & & & & $v \mid \mathrm{w}$ & \begin{tabular}{|l|l|}
$x$ & $y$
\end{tabular} & & & & @|\# \\
\hline 9 & & & & & & & & 1 & & & & & & & & & \\
\hline 10 & & & & & & & & \begin{tabular}{|l|l}
8 & 1
\end{tabular} & & & & & & & & & \\
\hline 11 & 2 & & & & & & & \begin{tabular}{|l|l}
52 \\
\end{tabular} & & & & & & & & & \\
\hline 12 & 2 & 3 & & & & & & 5 & 1 & & & & & & & & \\
\hline \begin{tabular}{|l|l|}
13 \\
\end{tabular} & & 1 & & & & & & \begin{tabular}{|l|l}
55 \\
\end{tabular} & 1 & & & & & & & & \\
\hline 14 & & & & & & & & 2 & 1 & & & & & & & & \\
\hline 15 & & 2 & 1 & & & & & & 2 & 1 & & & & & & & \\
\hline 16 & & 2 & 5 & & & & & & 1 & 1 & & & & & & & \\
\hline 17 & & & 2 & 1 & & & & 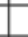 & & 4 & 2 & & & & & & \\
\hline \begin{tabular}{|c|}
18 \\
\end{tabular} & & & 2 & 4 & & & & & & 5 & 5 & & & & & & \\
\hline \begin{tabular}{|l|}
19 \\
\end{tabular} & & & & 3 & \begin{tabular}{|l|}
6 \\
\end{tabular} & & & & & 4 & 8 & 2 & & & & & \\
\hline 20 & & & & 4 & \begin{tabular}{|l|}
7 \\
\end{tabular} & & & & & 2 & 7 & & & & & & \\
\hline 21 & & & & & & 5 & & & & & & 7 & 3 & 1 & & & \\
\hline 22 & & & & & & 6 & & ] & & & & 5 & 52 & & 1 & & \\
\hline 23 & & & & & & 5 & 1 & 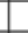 & & & & & \begin{tabular}{l|l|}
1 & 1 \\
\end{tabular} & & 1 & & \\
\hline 24 & & & & & & 2 & 5 & & & & & & & 2 & 1 & 2 & \\
\hline 25 & & & & & & & 2 & 5 & & & & & & 1 & 1 & 1 & \\
\hline 26 & & & & & & & 3 & 7) & & & & & & & 3 & & \\
\hline $27 \mid$ & & & & & & & 2 & 8 & & & & & & & 1 & & \\
\hline 28 & & & & & & & & \begin{tabular}{l|l}
3 & 2
\end{tabular} & & & & & & & & & \\
\hline \begin{tabular}{|l|}
29 \\
\end{tabular} & & & & & & & & & 2 & & & & & & & & \\
\hline 30 & & & & & & & & & 2 & & & & & & & & \\
\hline 31 & & & & & & & & & 2 & 1 & & & & & & & \\
\hline 32 & & & & & & & & & & 2 & & & & & & & \\
\hline 33 & & & & & & & & & & 2 & & & & & & & \\
\hline 34 & & & & & & & & & & & & & & & & & \\
\hline
\end{tabular}




\subsection{Análisis de los resultados}

Una vez recogidos los datos, los resultados se han plasmado en otra imagen que muestra, por colores, la densidad de usuarios que se han colocado en las distintas partes de la estación (Ver gráfico 8)

Gráfico 8: Resultado de la recogida datos en el que con colores más oscuros se muestran las zonas con mayor afluencia de público.

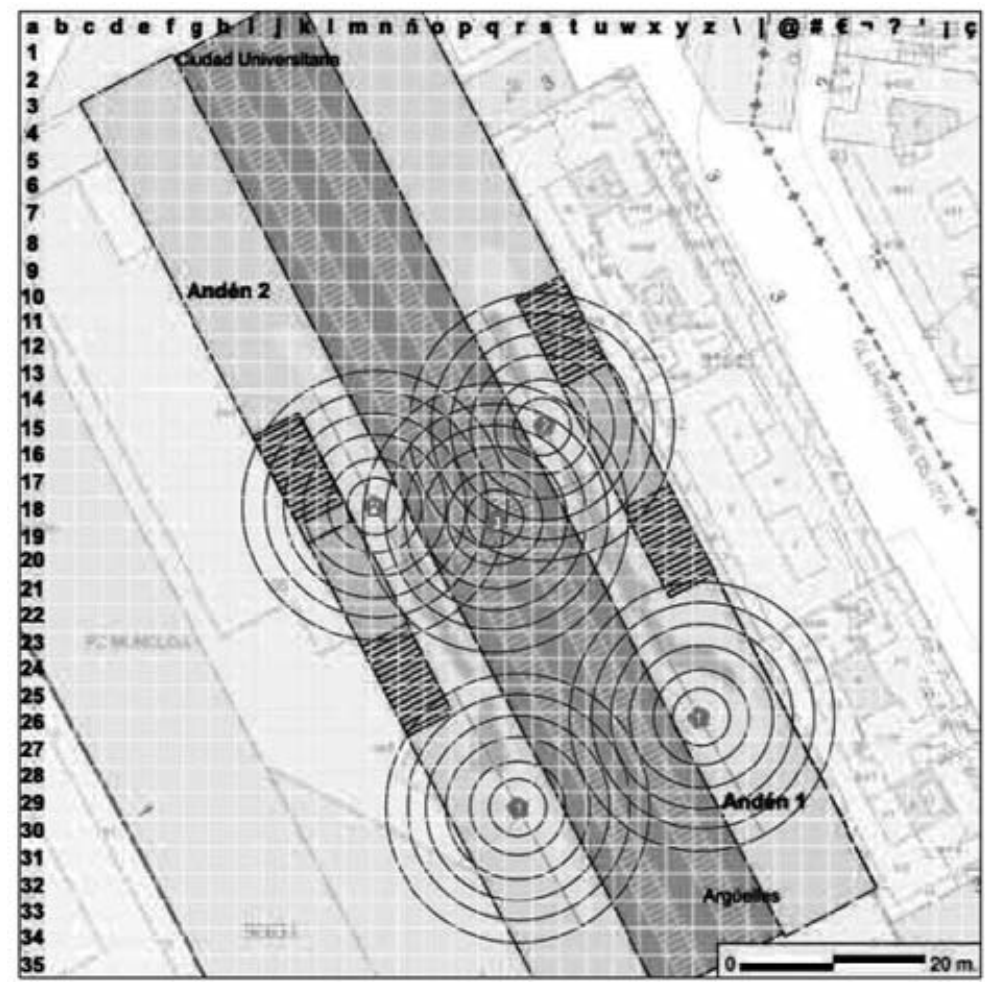

A partir de los datos obtenidos, podemos decir que las partes de la estación donde más se concentró el público durante el tiempo de la muestra fueron las correspondientes a todo el largo de la plataforma central. Asimismo, en un semicírculo alrededor de la pantalla 1 del andén 1 y a las centrales de la plataforma, más una pequeña cantidad cerca de la pantalla 1 del andén 2 . Más en concreto, las pantallas que más usuarios concentraron durante el tiempo de la muestra fueron la número 3 y la número 1 del Andén 1.

Por tanto y como conclusión importante, consideramos que las colocación de las pantallas sí obedece a una estrategia premeditada y que los responsables del canal han buscado provocar un efecto de conformidad entre los potenciales espectadores. En este sentido, las pantallas más consultadas han sido las situadas en los pies de las escaleras y en la parte central de la estación, donde abarcan un espectro de visión muy amplio. 


\section{Investigación sobre niveles sonoros en los ferrocarriles metropolitanos}

En el caso del sonido, y teniendo en cuenta que sólo la programación de Canal Metro incluye sonido, hemos tratado de medir el impacto del sonido en el devenir normal de ese canal en todas las horas de funcionamiento de la Línea 6, en la estación de Avenida de América del Metro de Madrid. En este caso, pretendíamos:

- Averiguar cuántas personas pasan por la estación en una semana

- Saber qué niveles de decibelios se registran en una semana dentro de la estación

- Calcular si los niveles de mayor ruido van parejos al de mayor afluencia de viajeros

- Evaluar si el nivel de ruido de la estación supera en algún momento el umbral de los 50 decibelios (contaminación acústica) y el de los 80 decibelios (umbral del dolor).

\subsection{Metodología}

El estudio se realizó entre el lunes 21 de marzo de 2005 y el domingo 29 de marzo de 2005, entre las 6 de la mañana y la 1,30 horas de la madrugada. Para ello, utilizamos cinco equipos de tres personas que hicieron turnos de cuatro horas.

Cuadro 2: Horarios de los turnos y lugar de recogida de datos

\begin{tabular}{|c|c|c|c|c|c|}
\hline \multicolumn{7}{|c|}{ Estación de Avenida de América - Línea 6 } \\
\hline Lugar & Turno 1 & Turno 2 & Turno 3 & Turno 4 & Turno 5 \\
\hline Duración muestra & 6:00-10:00 & 10:01-14:00 & 14:01-18:00 & 18:01-21:00 & 21:01-1:30 \\
\hline
\end{tabular}

Para lograr los objetivos marcados se realizaron dos tipos de recogidas de datos. Por un lado, se usó un contador de afluencia de público que utilizaron los tres miembros del equipo para ir anotando cada cinco minutos el número de personas que deambulaban por los tres andenes de la estación (que cuenta con un pasillo central).

Por otro lado, se utilizó un sonómetro PCE-322A de clase 2, que permite realizar mediciones generales en los trabajos de campo. Este sonómetro cuenta con una variante del micrófono de condensador, que utiliza el efecto duradero de una polarización electroestática a través de un electrodo laminar, no necesitando así alimentación alguna.

Los valores que se transmitieron a través de la interfaz digital se fueron guardando en las memorias del dispositivo para luego descargarlas en un ordenador portátil. Las tomas fueron automáticas, se hicieron cada cinco minutos en el amplio horario citado y se efectuaron desde el pasillo central.

\subsection{Análisis de los datos}

Tras siete días de recolección de datos, podemos manifestar que existe un fuerte comienzo de sesión en todos las jornadas laborales con una alta ocupación de los andenes hasta las 10:30 de la mañana. A partir de esta hora, existe una constante de ocupación baja hasta las 13:15 horas. Posteriormente se registra, una fuerte subida (incluso en los días del fin de semana) en el entorno de las 20:00 horas, a la que sigue una paulatina pérdida de pasajeros hasta que cierra el servicio.

Como es lógico, las cotas de ocupación de las pantallas coinciden con las horas de máximo uso de los transportes públicos urbanos en general. Asimismo, hay que sig- 
nificar que en los fines de semana la tendencia cambia con respecto a los días laborables. Aparte del cambio de hábito del sábado y el domingo, los viernes por la noche se rompe la caída en picado de usuarios que se registra en el resto de jornadas a partir de las 20:00 horas. El uso para ocio nocturno y la salida de muchas personas hacia las estaciones de tren, autobús o avión, hace que la densidad de usuarios aumente significativamente en ese arco horario (Ver Gráfico 9).

Gráfico 9: Comparativa semanal de la afluencia de viajeros en la estación

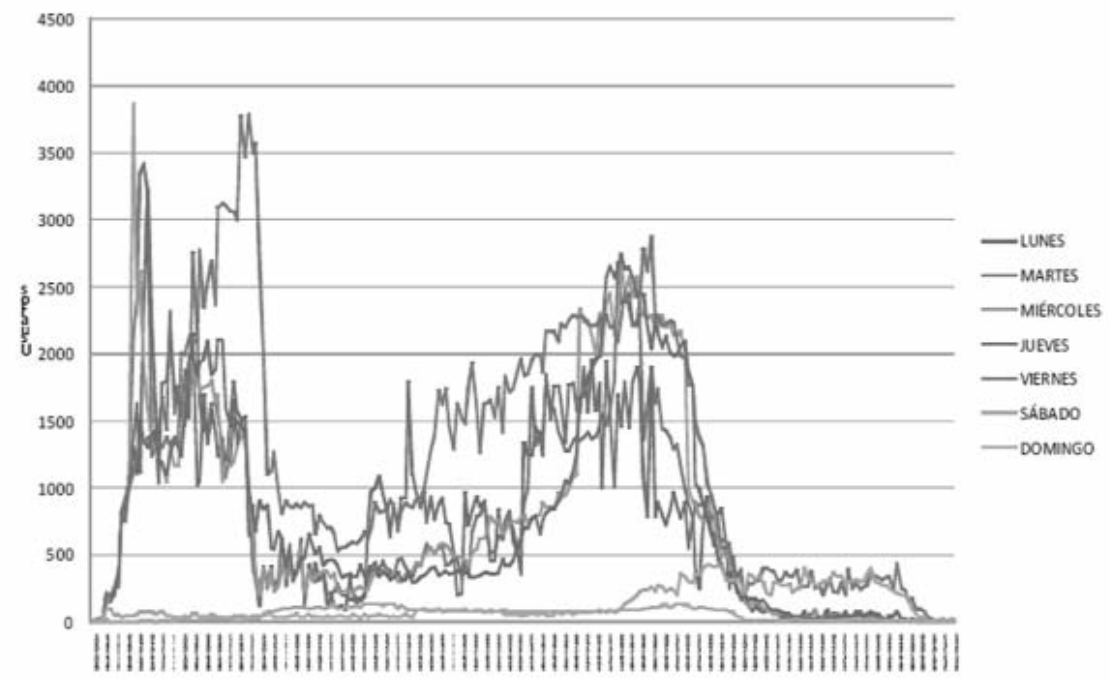

En lo que respecta a los decibelios registrados en los andenes de la estación durante esas jornadas, el lunes se registró contaminación acústica durante 635 minutos de la jornada, lo que supone un $54,27 \%$ del total del tiempo del servicio.

El martes se registró contaminación acústica durante 645 minutos de la jornada, lo que supone un $55,12 \%$ del total del tiempo del servicio.

El miércoles se registró contaminación acústica durante 421 minutos de la jornada, lo que supone un $35,98 \%$ del total del tiempo del servicio.

El jueves se registró contaminación acústica durante 360 minutos de la jornada, lo que supone un $30,76 \%$ del total del tiempo del servicio.

El viernes se registró contaminación acústica durante 681 minutos de la jornada, lo que supone un $58,20 \%$ del total del tiempo del servicio.

El sábado se registró contaminación acústica durante 89 minutos de la jornada, lo que supone un $7,60 \%$ del total del tiempo del servicio.

En resumen, en toda la semana, se superó el nivel de los 50 decibelios durante 3.136 minutos, lo que supone un $38,29 \%$ del total del tiempo del servicio. También se superó el nivel de los 80 decibelios durante 190 minutos, lo que supone un 2,31\% del total del tiempo del servicio (Ver Gráfico 10) 
Gráfico 10: Comparativa semanal del nivel sonoro los momentos en los que superó el nivel de los 80 decibelios

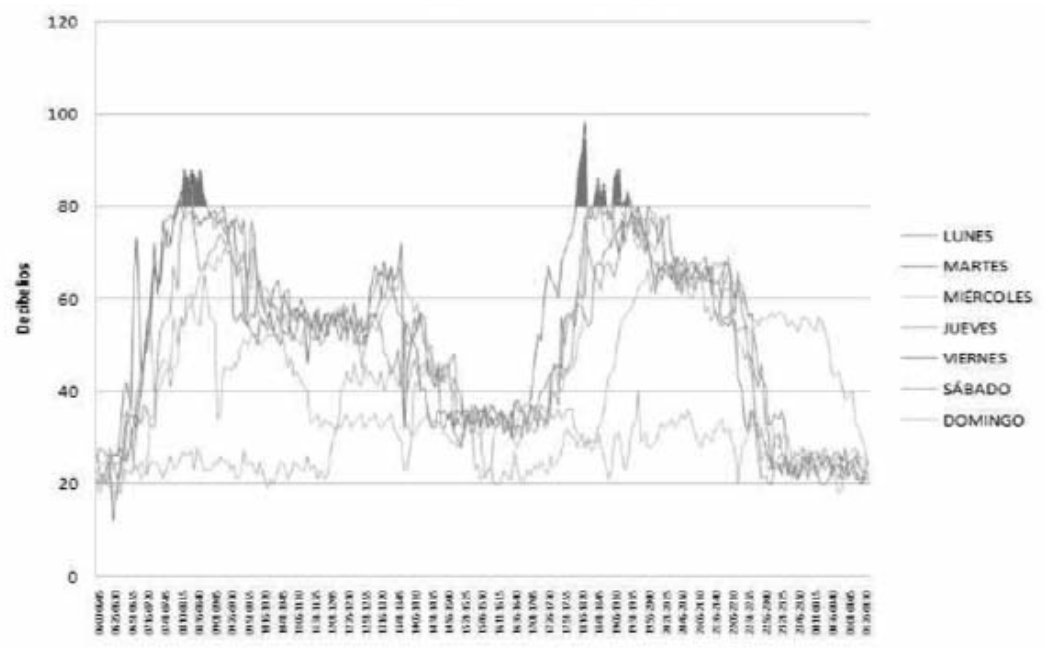

Podemos concluir, por tanto, que en más de un tercio del total del tiempo del servicio que se prestó en esos días, el ambiente de la estación y el de las propias pantallas, estuvo viciado con una contaminación acústica que superó las cotas del ruido soportable en más del 2 por ciento del total. En el cómputo global, estos datos están suavizados porque en los fines de semana la afluencia de pasajeros y la llegada y salida de convoyes disminuye. Este elevado porcentaje de contaminación acústica se puede achacar a varios factores:

- La concentración de personas

- El ruido producido por los propios convoyes al llegar y al salir de la estación

- El sonido de la programación de Canal Metro

\subsubsection{La interferencia del sonido}

A lo manifestado, hay que añadir que Canal Metro no comenzó siendo un medio de comunicación con sonido, sino que estaba basado en imágenes y en textos subtitulados. Con el tiempo, a esta configuración se le añadió el sonido de los locutores en modo "off". Pero este paso no ha sido del agrado de todos. Como también se ha podido ver, el nivel de ruido en los andenes supera el listón de lo permitido por las reglamentaciones. Y uno de los factores que provocan esta contaminación sonora es, precisamente, la inclusión del sonido de los locutores dentro de la programación.

En la encuesta realizada, un 72 por ciento de los entrevistados se mostraba en total disconformidad con que este tipo de canales "de acompañamiento" tuvieran sonido incorporado dentro de sus contenidos. De hecho, un gran número de usuarios manifiesta que eliminaría el sonido de la programación.

Quizá como solución, un punto intermedio podría ser habilitar una red de distribución del sonido dentro de las instalaciones del servicio (en este caso, el Metro), que funcionara a través de Wi-Fi y/o de radio. De esta manera, cualquier persona que dis- 
ponga de un terminal con estas tecnologías podría seguir el contenido de la programación con sonido sin perturbar al resto de pasajeros.

\section{Investigación sobre la respuesta de la audiencia}

En el amplio contexto de la investigación, la última parte la centramos en averiguar qué opina el público que usa estos medios de transporte con respecto a los canales multimedia tratados.

\subsection{Metodología}

Para conocer la respuesta de los usarios, realizamos un total de seis encuestas, divididas en dos áreas: una sobre Canal Metro y otra sobre Canal Bussi. Ambas fueron del tipo presencial mixta (cerrada y de respuesta múltiple) y abarcaba un total de 12 preguntas.

Las encuestas se realizaron, en el caso del metro madrileño, en la misma puerta de entrada de las estaciones de Moncloa, Príncipe Pío y Nuevos Ministerio. En el caso de los autobuses, en la parada de la calle Pintor Sorolla de Valencia, donde confluyen las líneas 4, 6, 8, 9, 10,11, 16, 26, 28, 31, 32, 36, 70, 71 y 81 de la EMT valenciana.

En Madrid se realizaron dos oleadas: el 20 de diciembre de 2004, en la que se entrevistaron a 203 personas, y el 20 de diciembre de 2006 y respondieron 207 personas. En Valencia, el 20 de diciembre de 2005 se realizaron 187 entrevistas y el 20 de diciembre de 2007 otras 204. De la misma manera, y junto a las encuestas presenciales, se abrió en Internet la posibilidad de responder a la misma encuesta a través de la dirección www.interrogantes.com. La web estuvo activa del 1 de abril de 2008 hasta el 5 de mayo de 2008 y recogió 48 respuestas sobre el Metro y 56 sobre el Autobús de Valencia.

Cuadro 3: Lugar y duración de las muestras de encuestas

\begin{tabular}{|c|c|c|c|c|c|c|}
\hline & \multicolumn{3}{|c|}{ Canal Metro } & \multicolumn{3}{c|}{ Canal Bussi } \\
\hline Lugar & Bocas & Bocas & Online & Parada & Parada & Online \\
\hline $\begin{array}{c}\text { Duración } \\
\text { muestra }\end{array}$ & $20-12-04$ & $20-12-06$ & $01-04-08$ & $20-12-05$ & $20-12-07$ & $01-04-08$ \\
\cline { 2 - 7 } & & & $05-05-08$ & & & $05-05-08$ \\
\hline
\end{tabular}

Las preguntas planteadas en el cuestionario de la encuestas fueron las siguientes:

1. ¿Cual es su edad?

2. ¿Cuál es su sexo?

3. ¿Cuál es su ocupación?

4. ¿Cuántas veces utiliza el Metro/Bus a lo largo de la semana?

5. ¿Cuánto suele esperar en el andén antes de que llegue el tren/bus?

6. ¿En la estación/línea de bus más próxima a su casa existe alguna pantalla?

7. ¿Suele mirar a esas pantallas mientras espera el tren/bus?

8. ¿Cree que el canal informativo es un valor añadido que hace más cómodo su viaje?

9. ¿Qué porcentaje de publicidad cree que se emite en la programación?

10. ¿Le gustaría que también hubiera pantallas dentro de los vagones/en las paradas? 
11. ¿Cree que la información que se ofrece tiende hacia alguna opción política en particular?

12. ¿Qué contenidos le gustaría que ofreciera la programación? (opción de respuesta múltiple)

13. ¿Considera que la calidad sonora es aceptable y que se entiende bien al locutor (solo metro)?

14. ¿Eliminaría/incorporaría el sonido de la programación?

15. ¿Cree que se efectúa un mantenimiento correcto de las pantallas?

\subsection{Análisis de resultados del Canal Metro}

Tras recoger y gestionar los datos, las encuestas arrojaron los siguientes resultados globales:

En el caso de Canal Metro, el perfil del público es mayoritariamente femenino, entre 16 y 41 años, estudiante y trabajadora por cuenta ajena. El resumen de datos manifiesta los siguientes resultados por parte de los encuestados:

- Usan el metro entre 11 y 20 veces a la semana.

- Esperan entre 2 y 5 minutos a que llegue el tren.

- Más de un $60 \%$ no tiene una pantalla en la estación más cercana a su casa.

- A veces las mira, piensa que es un valor añadido al servicio.

- Les gustaría que hubiera pantallas dentro de los vagones.

- Consideran que la programación emite mucha publicidad: entre un 50 y un $75 \%$ de la programación.

- Demandan más programas musicales, documentales y más deporte.

- Creen que la información que ofrecen las pantallas no tiende a ninguna ideología.

- Son críticos con la calidad sonora, mayoritariamente consideran que no es la correcta. Hay una buena parte de encuestados que eliminarían todo sonido de la programación. También consideran que el mantenimiento de los equipos es regular.

La mayoría de usuarios encuestados siguen habitualmente la programación de Canal Metro (Siempre: $42 \%$, A veces: $46 \%$ ). Consideran que Canal Metro es un valor añadido que hace más cómodo su transporte (51\%). Para este público, la publicidad siguen sin tener un buen grado de aceptación, a pesar de ser motor económico que facilita la creación de medios de comunicación como Canal Metro. El 36,6 \% de los encuestados tienen una consideración errónea de la cantidad de publicidad que se emite, pues la cifran entre un 50 y un 75 por ciento del total del contenido, cuando el peso de la publicidad real es del $20 \%$.

Una consideración importante es que los usuarios de Canal Metro entrevistados consideran, con el 63 por ciento del apoyo, que se debería ampliar la instalación de pantallas al interior de los vagones. Se considera, así, que el grado de aceptación del canal es tal que si, su programación también se emitiera en los propios trenes, sería bien recibido por los usuarios.

\subsubsection{Preferencias de los usuarios}

Cuando se les pregunta por sus preferencias y los contenidos que más les interesan de la programación, la respuesta responder a los siguientes parámetros: 
- Música, $56 \%$.

- Documentales: $45 \%$.

- Películas, 33\%.

- Series, $11 \%$.

- Deportes, $45 \%$.

- Dibujos Animados, 39\%.

- Concursos, $13 \%$.

- Reality/corazón, 3\%.

- Otros, 6\%.

Como se puede ver, la Música, los Documentales, y los Deportes son los temas más pedidos, con Dibujos Animados y Películas por detrás. Por otro lado y en relación con el grado de independencia y objetividad del canal, el 38\% de los encuestados responden que los contenidos no tienden hacia ninguna opción política en particular.

\subsection{Análisis de resultados del Canal Bussi}

En el caso de Canal Bussi de los autobuses, se aprecia que el público es mayoritariamente femenino, de entre 21 y 41 años, estudiante y trabajadora por cuenta ajena. Los encuestados completan perfil con lo siguientes parámetros:

- Usan el autobús entre 10 y 20 veces a la semana

- Esperan entre 2 y 10 minutos a que llegue a la parada.

- Más de un $60 \%$ sí toma una línea con coches que incorporan pantallas.

- La mayoría reconoce que mira las pantallas habitualmente y piensa que es un valor añadido al servicio.

- No les gustaría que hubiera pantallas en las paradas.

- Consideran que la programación emite un 50\% de publicidad.

- Echan en falta programas musicales y más deporte.

- Creen que los contenidos informativos que ofrecen no favorece ninguna tendencia ideológica.

- Mayoritariamente no incluirían sonido a la programación y consideran que el mantenimiento de los equipos es correcto.

En el caso del Canal Bussi, casi la mitad de los encuestados afirmó que sigue el canal mientras viaja (47\%). Según se deprende de los datos recogidos, casi uno de cada dos usuarios ven la programación habitualmente. La mitad de los encuestados considera que Canal Bussi es un aliciente para tomar el autobús en vez de otro tipo de transporte (49\%). En las ciudades donde Bussi se ha puesto en marcha en estos años (Valencia, Sevilla y San Sebastián), sólo la capital del Turia contaba con suburbano a la hora de realizar esta investigación.

Canal Bussi emitió un 23,6 por ciento de publicidad dentro de su programación total en la media de las investigaciones realizadas entre 2004 y 2007. Pero tal y como se ha visto anteriormente, una cosa es la estrategia de programación de las compañías de explotación de medios y otra la percepción que tienen las personas que visionan el canal. De hecho y como en el caso del canal de Metro, la mayoría de encuestados creen que el porcentaje de publicidad que se emite en los autobuses es bastante mayor del que indican los datos de seguimiento. 
En cuanto a sus propuestas de contenidos, los encuetados manifiestas las siguientes preferencias de programación:

- Música, 31\%

- Documentales: $16 \%$

- Películas, 7\%.

- Series, $10 \%$

- Deportes, $23 \%$.

- Dibujos Animados, 3\%.

- Concursos, $4 \%$.

- Reality/corazón, $1 \%$.

- Otros, $11 \%$.

Como se puede ver, la música, los deportes y los documentales vuelven a ser los contenidos que los usuarios prefieren. Por parte de los contenidos deportivos, no sería más que ampliar la información que se ofrece actualmente. El problema viene cuando se pide música, pues estamos hablando de un canal que no tiene sonido. ¿Estarán pidiendo los usuarios este servicio?

La independencia y la objetividad de los contenidos de Canal Bussi se reflejan en el sentir de los usuarios a tenor de las respuestas obtenidas. Es destacable que cerca de la mitad de los encuestados de esta encuesta respondiera que los contenidos del canal no tendían hacia ninguna opción política en particular (52\%). Se vuelven a dar las mismas circunstancias que en anterior caso de Canal Metro.

\section{Propuesta de modelo comunicacional para un canal en los transportes públicos}

Tras las investigaciones realizadas y habiendo analizado los elementos que han servido como base para esta publicación, proponemos un modelo comunicacional de ámbito restringido a todos los canales multimedia que operan u operarán en los transportes públicos como canales con programación propia, dinámica y actualizable.

Las características de este modelo parten de la definición de un canal global, permanente, integrador, complementario de los medios tradicionales y potenciador de la comunicación interna y externa. En conjunto, el posible modelo podría estar definido por los siguientes requisitos:

1. Contribuir a potenciar la capacidad y eficacia del sistema de transportes

2. Garantizar la accesibilidad a las emisiones, recursos informativos y utilidades del sistema

3. Crear una estructura estable y funcional que sirva para garantizar una vía rápida y eficaz de comunicación a los usuarios de transportes públicos

4. Poner a disposición del usuario contenido de información general y entretenimiento que le informen, hagan más agradable su viaje y fomente el uso del transporte público.

5. Acercar a los ciudadanos la realidad del transporte público (como alternativa eficaz ante otros medios de locomoción).

6. Reforzar la identificación de los usuarios con el medio de transporte.

7. Transmitir una imagen global de calidad en las actuaciones.

8. Fomentar el conocimiento de los servicios para maximizar su uso. 
9. Otorgar protagonismo a los equipamientos y recursos (técnicos, humanos y logísticos)

10. Reforzar la difusión de las campañas institucionales.

11. Humanizar y prestigiar el trabajo diario de los medios de transporte público.

12. Contribuir a la difusión de las actividades culturales, y favorecer los de integración de los ciudadanos de origen extranjero.

13. Comunicar el esfuerzo de gestión de la Administración en el ámbito del transporte.

14. Crear una red operativa con capacidad para acometer acciones urgentes en situaciones de crisis.

15. Facilitar el acceso de los ciudadanos a contenidos de utilidad, información y entretenimiento.

16. Incorporar una nueva y amplia oferta de contenidos y servicios de comunicación.

17. Identificar las diversas tecnologías disponibles y aplicarlas en función de los distintos objetivos y los diferentes públicos destinatarios.

18. Rentabilizar las posibilidades de los canales de comunicación gracias a las tecnologías digitales, adaptando los formatos, contenidos y mensajes a las necesidades y expectativas de los ciudadanos.

19. Reforzar la difusión de las campañas institucionales. Generar protocolos y metodologías para el diseño, producción y difusión de los contenidos y servicios de comunicación, simplificando los procesos, mejorando la eficacia y garantizando la rentabilidad y la sostenibilidad de los proyectos que se desarrollen.

20. Favorecer el carácter proactivo del modelo de comunicación (modelos tecnológicos de participación 2.0)

21. Asentar las posibilidades de interactividad, favoreciendo la participación de la ciudadanía también en los contenidos que se proponen.

Asimismo, y para lograr sus objetivos, todos los medios han de seguir unas estrategias basadas en una serie de parámetros, entre los que destacamos:

1. Se debe permitir la interactividad.

2. Por las especiales características del canal, las pautas de emisión deben ser diarias, semanales y mensuales y los temas de sus contenidos deben recoger, al menos, estos temas: Pasatiempos; Tiras de humor; Información general; Información deportiva; Información cultural; Información de turismo; Información de ocio; Información económica; Vídeos musicales; Trailers de películas; Animación; Cartelera de la ciudad; Curiosidades y Ofertas de empleo, entre otros.

3. Cualquier tipo de contenido grabado debe durar, como máximo, 45 segundos.

4. Se debe fomentar el uso de la ampliación de contenidos en la web.

5. Se deben crear elementos de apoyo al medio de comunicación basadas en tecnologías web.

6. Se deben crear contenidos Premium con beneficios en acciones de publicidad o promoción a los usuarios registrados en la página web.

7. Se debe fomentar la inmersión de los contenidos y la interactividad del medio en las redes sociales. 
8. Se debe limitar la emisión de publicidad.

9. Se debe diversificar la oferta de formatos de publicidad.

10. El uso de colores debe ser llamativo pero no estridente con especial atención a las superficies planas con pocos degradados.

11. Se debe diseñar la pantalla de emisión de los contenidos dividiendo la misma en tres partes:

- Una superior izquierda que ocupe $2 / 3$ de una división imaginaria de la pantalla en forma tanto vertical como horizontal que contenga los contenidos audiovisuales.

- Una inferior que ocupe 1/3 de una división imaginaria de la pantalla en forma horizontal que contenga las frases de los subtítulos de los contenidos.

- Una superior derecha que ocupe 1/3 de una división imaginaria de la pantalla en forma vertical que contenga datos complementarios.

12. La programación debe ser silenciosa, emitiendo el contenido de audio a través de la frecuencia de radio.

13. Se debe dar importancia, dentro de la programación, a los contenidos de servicio público.

14. Se debe dar importancia, dentro de la programación, a la información.

15. Se deben respetar las limitaciones de tipos de contenidos y horarios previstos por la ley, en especial a los que amparan al público

16. Se debe crear un manual deontológico y un código de autorregulación en el equipo de redacción.

17. Todas las piezas grabadas deben ser leídas y subtituladas.

18. La programación del tipo lineal cerrada en la parrilla debe seguir los parámetros del estándar de escaleta de la televisión convencional.

19. Se debe emitir la programación del canal en audio a través de una emisora de FM o AM en un radio limitado a los andenes y pasillos de la red de metro y en los vagones y coches de las redes de metro y autobús.

20. La programación debe permitir tanto emisiones grabadas como programas en directo.

21. La programación se debe emitir en pantallas terminales.

22. Se debe emitir la programación a través de otros terminales.

23. Se deben crear las herramientas concretas para dar accesibilidad y usabilidad a todo tipo de usuario, poniendo especial atención al colectivo de personas discapacitadas.

24. Se debe emitir en variedad de idiomas.

25. Se debe implantar en las pantallas del interior de los vagones y de los coches de un sistema paralelo de información de cada una de las estaciones en las que se realiza parada.

\section{Conclusiones}

Después de haber realizado las consiguientes investigaciones, las cuestiones planteadas que han servido como punto de partida, quedan de la siguiente forma: 
Ante la hipótesis "Los canales multimedia de los transportes públicos basan su estructura y su programación en los medios de comunicación tradicionales", se puede afirmar que sí se cumple. Queda demostrado por el análisis realizado a los elementos gráficos y a la programación que su cercanía con la televisión es importante tanto en su continente como en su contenido.

Ante la hipótesis "Los usuarios de los medios de transporte son favorables a la existencia y desarrollo de estos canales", se puede afirmar que sí se cumple. Queda demostrado por las respuestas dadas por los usuarios en las encuestas que creen que estos canales multimedia son valores añadidos al transporte público.

Ante la hipótesis "Los usuarios prefieren que no emitan sonido y que no sean intrusivos", se puede afirmar que sí se cumple. Queda demostrado por las respuestas dadas por los usuarios en las encuestas y por los altos niveles de contaminación acústica mencionados que prefieren un canal silencioso como es Canal Bussi.

Ante la hipótesis "La accesibilidad y usabilidad de estos medios no es completa al no amparar en su naturaleza el acceso a los contenidos de los minusválidos visuales" se puede afirmar que no se cumple. Queda demostrado por el análisis realizado a los elementos gráficos que el hecho de que Canal Bussi sea mudo impide a este colectivo acceder a sus contenidos. Por el estudio de niveles sonoros, Canal Metro tampoco es accesible porque existe en numerosas ocasiones tal contaminación acústica que lo hace inaudible.

\section{Referencias bibliográficas}

ASCH, Solomon E. (1956): "Studies of Independence and Conformity: A minority of one against a unanimous majority", en Psycology Monographs $n^{\circ} 70$. Nueva York, whole no. 416, pp. 1-70.

BASSAT, Lluís (2001): El libro rojo de la publicidad. Barcelona, DeBolsillo.

BURGUELIN, Olivier (1974): La comunicación de masas. Barcelona, Editions Planete y A.T.E.

CORTINA, E. (2004): "Extender el Canal Bussi a Madrid y Barcelona es nuestro primer objetivo", en Diario Las Provincias, 4 de julio, separata Euros, pp.E5. http://www.tmb.net/publicacions_tmb/inf2000/cast/5/57.jsp. [Fecha de consulta: 30 de noviembre de 2009]

SMITH, Eliot R. y MACKIE, Diane M. (1997): Psicología social. Madrid, Editorial Médica Panamericana.

VV.AA. (2003): Directiva 2003/10/CE de 6 de febrero de 2003 sobre las disposiciones mínimas de seguridad y de salud relativas a la exposición de los trabajadores a los riesgos derivados de agentes físicos (ruido). Estrasburgo, Parlamento Europeo.

VV.AA. (2006): "Servicios complementarios en las estaciones “, en TMB Transports Metropolitans de Barcelona

VV.AA. (2009): "SECTRA. Secretaría Interministerial de Planificación de Transporte de Chile", en Sectra: http://www.sectra.cl [Fecha de consulta: 9 de diciembre de 2009] 